\title{
Fundamentos para una metodología de diagnóstico del síndrome del ojo seco basado en la termografía infrarroja
}

\author{
Luis Enrique Llamosaㄹ, Jeimy Melissa Jaime-Díaz², Mariney Rodríguez Rengifo-Rincón ${ }^{3}$. \\ Profesor titular - Facultad de Ciencias Básicas, Universidad Tecnológica de Pereira \\ Ingeniera Física, Joven Investigadora 2015-2016 COLCIENCIAS, Universidad Tecnológica de Pereira \\ 3. Oftalmóloga, Docente - Programa de Medicina - UNILIBRE de Cali
}

Fecha de Recepción: 29/5/2015

Fecha de Evaluación: 19/9/2015

Fecha de Solicitud de Correcciones: 11/2/2016

Fecha de Aceptación: 23/2/2016

\section{Resumen:}

El Síndrome del ojo seco afecta aproximadamente el $40 \%$ de la población, si no se trata a tiempo puede llegar a causar trastornos en la salud visual de las personas. En este trabajo se presentan los fundamentos para el diseño y la implementación de una metodología para el diagnóstico de esta enfermedad, la cual se basa en la variación de la temperatura de la superficie ocular de sujetos normales comparados con pacientes con ojo seco, utilizando la técnica de termografía infrarroja. Este protocolo se fundamenta en el cambio de la temperatura de la córnea después de mantener los ojos abiertos sin parpadeo durante un tiempo determinado, seguida de la disminución de la misma en una etapa de parpadeo durante 30 segundos. De acuerdo a los resultados obtenidos para una muestra de 40 individuos, 20 de ellos con Síndrome del ojo seco, se determinó que el comportamiento de la variación de la temperatura ocular entre sujetos sanos y con ojo seco difiere significativamente en lo que respecta a la tasa de disminución de la temperatura de la córnea con cada parpadeo, lo que permite fundamentar la metodología propuesta utilizando la termografía infrarroja como una herramienta de diagnóstico cuantitativa y no invasiva para el estudio de esta enfermedad.

Palabras clave: diagnóstico, metodología, oftalmopatías, termografía. (Fuente: DeCS)
Fundamentals for a methodology of diagnosis of the dry eye syndrome based in infrared thermography

\begin{abstract}
The dry eye Syndrome affects approximately $40 \%$ of the population, if not treated in time can cause health disorders in the visual health of people. This paper presents the basis for the design and implementation of a methodology for the diagnosis of this disease, which is based on the variation of the temperature of the ocular surface of normal subjects compared with patients with dry eye, using the infrared thermography technique. This protocol is based on the temperature change of the cornea after keeping open eyes without flashing for a certain time, followed by the reduction of the same, in a stage of blinking for 30 seconds. According to the results obtained for a sample of 40 individuals, including 20 with dry eye Syndrome, it was determined that the behavior of the variation of eye temperature between normal subjects and with dry eye, differs significantly with respect to the rate of decrease of the temperature of the cornea with each blink, allowing support of the proposed methodology using infrared thermography as a quantitative tool for the noninvasive diagnosis and study of this disease.
\end{abstract}

Keywords: diagnostic, methodology, eye diseases, thermography.

\section{Introducción}

La ceguera o mala visión afecta a millones de personas. Las principales causas de la mala visión son la catarata, degeneración macular y la retinopatía diabética $(1,2)$. El síndrome del ojo seco (SOS) no es una de las principales causas de la discapacidad visual, pero es la más común de las afecciones oculares, y si no se trata a tiempo puede llegar a causar mayores trastornos en la superficie ocular. Aunque el 75\% de las personas mayores de 60 años sufren el síndrome del ojo seco, éste también está afectando a la población juvenil, cuando se realizan actividades visuales agotadoras, tales como lectura mantenida, exceso de televisión o el uso del computador largas horas del día, se produce resecamiento en la superficie ocular (3). El parpadeo es responsable de distribuir la lágrima uniformemente sobre la película lagrimal, mientras se está frente a un monitor se reduce en un $50 \%$, por lo tanto desencadena poca producción de las lágrimas; además, otros factores como la contaminación ambiental, las condiciones de mucho sol, humo y aire seco incrementan el riesgo de sufrir de ojo seco (4). En la actualidad, el estudio de la sequedad ocular ocupa un lugar privilegiado en las investigaciones oftalmológicas, ya que los intensos esfuerzos destinados al estudio de esta singular enfermedad están orientados principalmente hacia los aspectos relacionados con su fisiopatología y el desarrollo de nuevos y efectivos medicamentos (3); por lo que se ha dejado atrás el estudio de nuevas opciones de diagnóstico totalmente cuantitativas y no invasivas. Generalmente el diagnóstico de ojo seco se basa en el desarrollo de dos pasos, la recopilación de datos a partir de las manifestaciones que el enfermo refiere, y los test de diagnóstico: Schirmer I, tiempo de ruptura de la película lagrimal (BUT), tinción con rosa de Bengala, fluoresceína o lisamina verde $(3,5,6)$, aunque estas pruebas son utilizadas por los oftalmólogos actualmente, no son totalmente confiables ya que arrojan resultados no muy precisos y además son muy incomodas para el paciente.

La termografía biomédica no es invasiva, no es ionizante, libre de riesgo, amigable con el paciente y el costo es considerablemente bajo (7). Distribuciones de temperatura de la superficie de la piel plasmadas en una imagen térmica se pueden utilizar para identificar las áreas de anormalidad térmica, para la detección temprana y monitoreo de los procesos de enfermedad; y para controlar la eficacia de los procedimientos de tratamiento (8). Estas características, hacen que esta técnica sea una herramienta efectiva de diagnóstico, respaldada por treinta años de uso clínico y más de ocho mil estudios arbitrarios en publicaciones médicas como una alternativa segura y eficiente en la exanimación del cuerpo humano, pues las imágenes térmicas de la piel humana han sido utilizadas para controlar la distribución de la temperatura de la misma (9). Los cambios característicos en los patrones normales del cuerpo se asocian a diferentes fenómenos patológicos dando la pauta para llevar a cabo investigaciones 
no invasivas que resulten valiosas para el diagnóstico. Anomalías como la inflamación y la infección consecuencia de tumores malignos, se localizan por aumentos de temperatura que se muestras como puntos calientes o patrones en una imagen térmica (10). A pesar de que no es específica, la termografía infrarroja es un potente detector de problemas que afectan la fisiología de un paciente. Si las imágenes térmicas son capturadas bajo parámetros específicos que pueden influir como la edad, tomas en horas del día adecuadas y condiciones ambientales controladas (11), pueden ser interpretadas fácilmente para diagnosticar ciertas condiciones y para controlar la reacción de la fisiología de un paciente a las tensiones térmicas y otros. Algunas de las principales áreas donde se utiliza la termografía infrarroja con éxito son la neurología, perturbaciones vasculares, enfermedades reumáticas, lesiones de tejidos blandos (10), oncología (especialmente cáncer de mama) (12), trastornos dermatológicos, problemas en el sistema nervioso, neonatal, cirugía y por supuesto trastornos oculares $(9,13-15)$. Hasta el momento son muy pocos los estudios que se han realizado en el ojo seco utilizando termografía biomédica. Fujishima et al., evaluaron la temperatura de la superficie ocular de individuos japoneses con ojo seco y sujetos normales. Los sujetos mantuvieron los ojos abiertos durante diez segundos parpadeando y se midió cada segundo la temperatura de la córnea. El cambio de la temperatura de la córnea en los pacientes con ojo seco fue de $-0,21{ }^{\circ} \mathrm{C}$, mientras que en los sujetos normales $-0,61^{\circ} \mathrm{C}$. Este estudio demostró la utilidad de la termografía por radiación infrarroja en la evaluación de pacientes con ojo seco, ya que se encontró que en sujetos con ojo seco la tasa de disminución de la temperatura de la córnea fue menor. Los autores afirman que la técnica es "fácil" y puede llegar a ser un procedimiento eficaz para el diagnóstico clínico de ojo seco, además de proporcionar información adicional sobre la dinámica lagrimal de la superficie ocular en relación con el parpadeo (5). Tai Yuan Su et al., desarrollaron a partir de la termografía infrarroja (IR) sin contacto un protocolo para medir la variación temporal de la temperatura de la superficie ocular de sujetos sanos y sujetos con ojo seco durante un período de 6 segundos. Los resultados de este estudio sugieren que las imágenes térmicas puede ser utilizadas como una herramienta eficaz para la detección sin contacto del ojo seco, sin embargo un mayor desarrollo de este protocolo puede dar lugar a la aplicación de un sistema de diagnóstico sin contacto de ojo seco que puede aliviar la incomodidad y molestias que el paciente experimenta en el diagnóstico clínico del ojo seco convencional (16).

Síndrome del ojo seco: Las lágrimas sirven para proteger los ojos y mantenerlos lubricados. El Síndrome de Ojo Seco (SOS), también conocido como queratitis sicca es una reducción en la cantidad y/o calidad de lágrimas producidas. Esto sucede cuando las glándulas lacrimales localizadas en los párpados superior e inferior no producen suficientes lágrimas o no producen lágrimas de la calidad adecuada. Este fenómeno causa irritación, escozor, rasquiña, enrojecimiento e incomodidad $(4,17,18)$. La lágrima tiene tres fracciones diferentes, que se producen en glándulas distintas: Las glándulas lagrimales segregan la fracción acuosa, que constituye el $99 \%$ del total de la lágrima. La conjuntiva, que tapiza la esclerótica (parte blanca del ojo) y la pared posterior de los párpados, está compuesta por células calciformes que producen mucina, la cual constituye tan solo el $0.5 \%$ del total lagrimal. Además los párpados tienen en su espesor las glándulas de Meibomio, (que cuando enferman producen los orzuelos) las cuales producen grasa en bajas cantidades. Además la lágrima presenta un contenido en proteínas, enzimas e inmunoglobulinas, sustancias fundamentales ante determinadas enfermedades e infecciones que sufren los ojos cuando disminuyen los componentes de la lágrima $(4,19-22)$. Con base en lo anterior se plantean las siguientes preguntas de investigación: ¿Cuál sería la metodología experimental que debería utilizarse para realizar un protocolo de diagnóstico a partir de la técnica de la fotografía termográfica en sujetos que padecen el síndrome del ojo seco? ¿Cuáles son las diferencias termográficas entre personas que padecen el síndrome del ojo seco y personas que no lo padecen? Para este trabajo se planteó como objetivo general: Diseñar e implementar un protocolo para el diagnóstico del síndrome del ojo seco a partir la técnica de la fotografía termográfica.

\section{Materiales y métodos}

Se realizó un estudio experimental con grupo control, en el que la variable dependiente es la variación de la temperatura de la superficie ocular en la etapa de "fijación con parpadeo" que será descrita y definida más adelante en este artículo. La variable independiente es el grado de resequedad del ojo humano que se plantea indagar con base en el método diseñado, utilizando la técnica de la fotografía infrarroja, mediante la cual se analiza en intervalos de tiempo determinados, el comportamiento de la temperatura promedio de la superficie ocular de pacientes con ojo seco y sujetos sanos, en situaciones de fijación con y sin parpadeo. En la Figura 1 se muestra el equipo de medición y el montaje experimental con uno de los individuos que participaron en el estudio mientras se le realizaban algunas medidas.

Equipo de Medición: La cámara termográfica Fluke Ti32 es un procesador de imágenes térmicas que puede capturar de forma rápida y sencilla imágenes de gran calidad. El rango de medición de temperatura comienza en los $-20^{\circ} \mathrm{C}$ y asciende hasta los $600^{\circ} \mathrm{C}$. Cuenta con tecnología IR-Fusion ${ }^{\oplus}$, donde las imágenes térmicas y visuales pueden presentarse simultáneamente como una imagen térmica completa o como una imagen dentro de otra en distintos modos de combinación. Las imágenes térmicas se pueden analizar por medio del software SmartView y se pueden realizar informes a partir de las imágenes térmicas guardadas. La cámara termográfica proporciona funciones como: corrección de la emisividad, compensación de la temperatura reflejada de fondo y corrección de transmisión. Además ofrece paletas y modos de paletas especiales para mejorar y resaltar diferencias térmicas muy pequeñas en un escenario. La resolución de indicación de temperatura de este instrumento es de $0,01\left({ }^{\circ} \mathrm{F} \mathrm{o}{ }^{\circ} \mathrm{C}\right) \mathrm{y}$ el fabricante reporta una precisión $\pm 2 \% \mathrm{o} \pm 2{ }^{\circ} \mathrm{C}(23,24)$.

Figura 1. Explicación gráfica del protocolo experimental diseñado e implementado, se puede observar el equipo utilizado en el estudio.

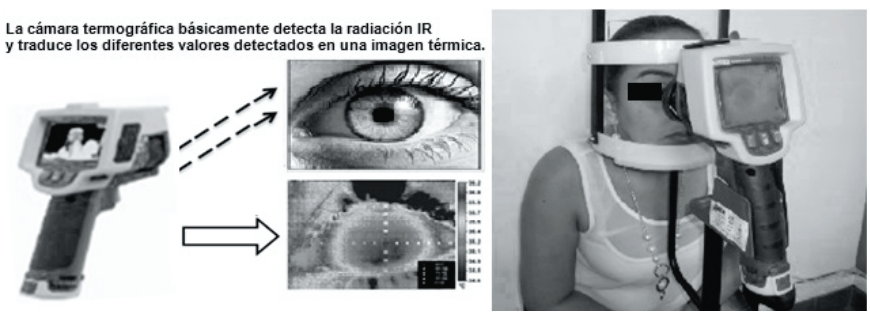

Protocolo de medición para la toma de las imágenes térmicas: Para implementar el protocolo se realizó un estudio, en el cual se seleccionaron 40 personas, 20 sujetos que manifestaron haber sido diagnosticados con síndrome del ojo seco por medio de una de las pruebas implementadas por los oftalmólogos en la actualidad; entre las que se pueden encontrar: las pruebas Schirmer I (la más utilizada), Tiempo de Ruptura de la película lagrimal (BUT), Tinción con Rosa de Bengala, Tinción con Fluoresceína y/o Test Rojo Fenol; y 20 sujetos normales; esta muestra poblacional se ubicó entre el personal estudiantil, administrativo y docente de la Universidad Tecnológica de Pereira. Se tuvo en cuenta que los participantes contaran con 
buena salud, los sujetos con fiebre o antecedentes de migraña no se incluyeron en este estudio, al igual que pacientes con enfermedades vasculares sistemáticas como la hipertensión y diabetes ya que presentan una mayor elevación térmica del ojo por la taza de flujo de sangre en la retina y la coroides (25). A la hora de realizar las pruebas, los sujetos de sexo femenino debían tener la cara sin maquillaje, ya que la aplicación de cosméticos puede cambiar la emisividad de la piel al momento de realizar la toma de las fotografías térmicas $(26,27)$. Los sujetos que usan habitualmente lágrimas lubricantes artificiales, no las usaron desde una hora antes de realizar las pruebas, esto con el objetivo de que la película lagrimal estuviera en condiciones reales. A pesar de que la técnica de la termografía IR aplicada en seres humanos es no invasiva, se aplicó teniendo en cuenta consideraciones de tipo bioético a partir de las recomendaciones de la declaración de Helsinki de 1964 y la Resolución No 008430 de 1993 del Ministerio de Salud de la República de Colombia con respecto a los principios éticos para las investigaciones médicas en seres humanos. Antes de realizar las mediciones cada sujeto fue informado sobre el objetivo del experimento y se le explicó claramente el procedimiento para participar en el estudio de investigación experimental, posteriormente diligenció la carta de consentimiento informado, donde aceptaba la participación en el estudio y las condiciones para llevarlo a cabo $(28,29)$. Para el estudio se tuvieron en cuenta problemas visuales como: miopía, astigmatismo y/o presbicia; para lo cual cada sujeto diligenció una rigurosa encuesta epidemiológica donde se evaluaron los signos y síntomas del síndrome del ojo seco, como el enrojecimiento, escozor, sensación de cuerpo extraño, sensación de arena, sensibilidad a la luz, ojos llorosos, ojos cansados, incomodidad con lentes de contacto, entre otros síntomas $(18,30)$; además se consultó que tipos de procedimientos quirúrgicos se le habían realizado, ya que una de las causas del ojo seco son las cirugías oftalmológicas que utilizan laser; también se tuvo en cuenta si el sujeto en sus labores cotidianas estaba expuesto continuamente a factores ambientales que pueden afectar la lubricación adecuada de la película lagrimal y se le consultó si sufría del Síndrome de Sjögren, otra causa de la resequedad en el ojo humano.

Se estableció que el entorno de medición: Laboratorio de Electrofisiología, ubicado en las instalaciones de la Facultad de Ciencias de la Salud de la Universidad Tecnológica de Pereira contara con las condiciones adecuadas para la toma de imágenes térmicas, teniendo en cuenta factores como la temperatura, la humedad relativa, las corrientes de aire, la luz y la polución (27); por lo tanto fueron monitoreadas y controladas las variables de humedad relativa de $50 \%(-10 \% y+20 \%)-$ "humedad relativa de confort" y temperatura ambiente de $21^{\circ} \mathrm{C}\left(-3{ }^{\circ} \mathrm{C} \mathrm{y}+3{ }^{\circ} \mathrm{C}\right)-$ "temperatura de confort", de tal manera que estas variables estuvieran en el mismo rango para todas las personas que conformaron la muestra (31), además al recinto se le adecuó la intensidad luminosa para tareas visuales simples con el objetivo de que no molestara a los participantes (200 - 300 Lux) (32); y se llevaron a cabo las mediciones durante el horario de trabajo del laboratorio, 8 a.m. - 5 p.m. Cada individuo estuvo con 10 minutos de anticipación en la habitación en que se tomaron las medidas antes de comenzar con la toma de las fotografías termográficas, tiempo necesario para una adaptación adecuada a la temperatura y humedad relativa del lugar, lo cual permitió una estabilización en la temperatura de la superficie ocular $(14,31,33,34)$.

Se tomaron las termografías IR de la superficie ocular y se realizó el análisis de las mismas, a los 40 participantes del protocolo experimental, de manera aleatoria con respecto a los sujetos participantes, sin conocimiento previo del grupo a que pertenecía cada sujeto de estudio por parte de las personas que tomaban los datos y realizaban el análisis. Las imágenes se tomaron inicialmente en el ojo derecho y luego en el ojo izquierdo. Para iniciar el procedimiento, la frente y la barbilla de los participantes fueron ubicadas contra un soporte de medición, de manera que el centro geométrico de la córnea estaba alineado con la cámara termográfica a una distancia de $7,5 \mathrm{~cm}$, como se ilustra en la Figura 1. Inmediatamente, tanto los sujetos sanos como los sujetos con ojo seco, permanecieron con la posición primaria de mirada durante 5 minutos, la cual consiste en una posición de mirada al frente, con el parpadeo normal; esta visión inicial se utiliza para que los ojos de los participantes se adapten a la temperatura, humedad e intensidad de la luz de la habitación en la posición en que se realizan las pruebas. Luego tuvieron una etapa de descanso con los ojos en reposo; es decir, cerrados durante 10 segundos. Posteriormente los sujetos mantuvieron los ojos en posición de fijación sin parpadeo durante 30 segundos; en este tiempo se hacen mediciones de la temperatura de la superficie ocular mediante la toma de imágenes termográficas, aproximadamente cada 2 segundos, ya que este es el tiempo que la cámara termográfica Ti32 se tarda para tomar y procesar la imagen térmica; finalmente los ojos estuvieron en posición de fijación con parpadeo normal en la cual se tomaron imágenes termográficas durante otros 30 segundos, en intervalos de 2 segundos, como se muestra en el cuadro 1 (5).

Cuadro 1. Distribución de tiempos para la toma de medidas con la cámara termográfica Ti32.

\begin{tabular}{cc}
\hline Posición Sujeto & Tiempo \\
\hline $\begin{array}{c}\text { Posición primaria de mirada } \\
\text { (Parpadeo normal) } \\
\text { Descanso } \\
\text { (Ojos cerrados) } \\
\text { Posición de fijación }\end{array}$ & 5 Minutos \\
(Ojos abiertos sin parpadeo) & 10 Segundos \\
Posición de fijación & 30 Segundos \\
(Ojos abiertos con parpadeo) & (Se toman las imágenes mínimo cada 2 segundos) \\
& (Se toman las imágenes minimimo cada 2 segundos) \\
\hline
\end{tabular}

Para la implementación del protocolo experimental diseñado, se realizó de manera inicial el análisis de cada imagen térmica por medio del software de la cámara termográfica Ti32: "SmartView 3.5", determinando la temperatura promedio de la región de la superficie ocular a analizar (superficie de la córnea); se analizaron los datos de toda la muestra poblacional a través del software para análisis de datos OriginLab 8.6. Es importante anotar que en el análisis de cada termograma se tomó un número de datos de temperatura que correspondían al número de los pixeles correspondientes a la región de la fotografía termográfica que se quería analizar, con base en ellos se obtuvo el valor medio de la misma en la región de la superficie ocular analizada, así como la desviación estándar y la varianza de los datos de temperatura analizados; en la figura 2 se ilustra el proceso. 
Figura 2. Imágenes térmicas del ojo derecho de uno de los individuos en los momentos de fijación sin parpadeo y de fijación con parpadeo, obsérvese la diferencia de temperaturas.

Fuente: Software de la cámara termográfica Ti32, SmartView 3,5.

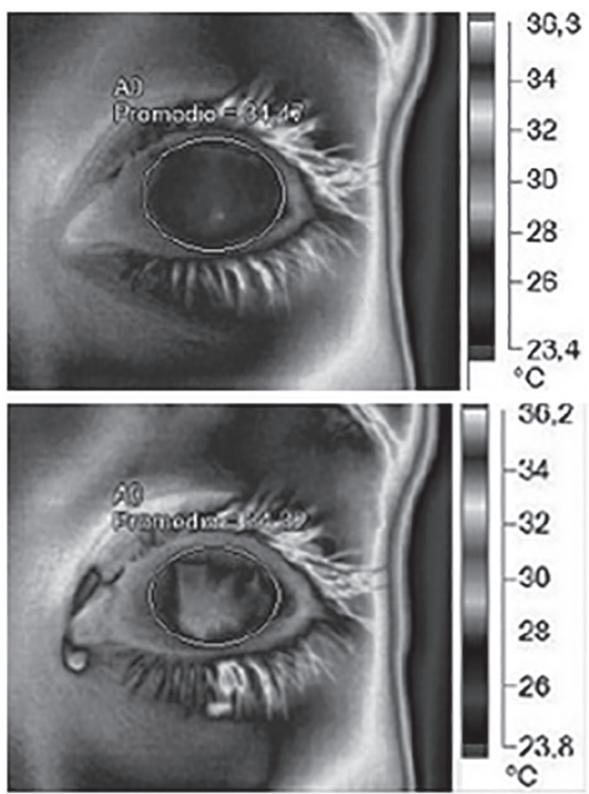

Para el análisis estadístico se tomaron los datos correspondientes a la variación de temperatura experimentada por lo sujetos sanos y con SOS en la posición de fijación con parpadeo; se verificó la normalidad de los datos y con base en los resultados de la prueba de normalidad de los mismos se decidió realizar la prueba U de Mann Whitney, prueba no paramétrica, mediante la cual se identifican diferencias estadísticamente significativas entre dos poblaciones, basada en el análisis de dos muestras independientes. Con base en la aplicación de esta prueba se quería demostrar que el método permitía establecer diferencias estadísticamente significativas entre la población con ojos sanos y la población diagnosticada con síndrome del ojo seco (SOS).

\section{Resultados}

Como ya se mencionó la muestra poblacional de estudio estuvo conformada por 40 participantes, 20 sujetos que manifestaron haber sido diagnosticados con síndrome del ojo seco por medio de una de las pruebas implementadas actualmente y 20 sujetos sanos. La muestra total incluyó 21 mujeres (52,5\%) y 19 hombres (47,5\%). En el cuadro 2 se específica el número de participantes a partir de rangos de edades establecidos $(1,20)$.

Cuadro 2. Distribución por edad y sexo de las personas evaluadas

\begin{tabular}{lcccccc}
\hline \multicolumn{1}{c}{ Rango de edades } & No. de hombres & $\%$ & No. de mujeres & $\%$ & Total & $\%$ \\
\hline Jóvenes (15-22 años) & 7 & 53,85 & 6 & 46,15 & 13 & 32,50 \\
Mediana Edad (23-40 años) & 4 & 28,57 & 10 & 71,43 & 14 & 35,00 \\
Edad Adulta (41-65 años) & 8 & 61,54 & 5 & 38,46 & 13 & 32,50 \\
\hline
\end{tabular}

Luego de analizar los datos obtenidos a partir de las imágenes termográficas se hizo la clasificación de los individuos según los resultados de la encuesta epidemiológica que cada uno de ellos diligenció antes de iniciar las pruebas; esto para comparar los resultados de las imágenes térmicas con los resultados de las encuestas, y así validar el protocolo formulado. Los resultados obtenidos presentan un cambio de la temperatura de la córnea después de mantener los ojos abiertos durante un tiempo determinado y una disminución de esta temperatura con cada parpadeo; siendo el comportamiento de la variación de la temperatura ocular en los sujetos con síndrome del ojo seco diferente con respecto a los sujetos sanos. Analizando los datos de cada individuo que participó en el estudio se notó que los cambios significativos entre un individuo con ojo seco y uno normal se muestran en la variación de la temperatura de la superficie ocular, con los ojos en posición de fijación con el parpadeo normal, por lo que se hizo un análisis riguroso de los datos en este punto. Examinando los cuadros y las gráficas obtenidas se tiene, que para la posición de fijación con parpadeo, para uno de los sujetos normales elegido al azar, la temperatura del ojo derecho disminuyó de $35,09^{\circ} \mathrm{C}$ a $34,75^{\circ} \mathrm{C}$ (Cuadro 3 y Figura 3), mientras que para el ojo izquierdo disminuyo de $34,81^{\circ} \mathrm{C}$ a $34,52{ }^{\circ} \mathrm{C}$ (Cuadro 3 y Figura 4). Para uno de los sujetos con síndrome del ojo seco elegido también al azar, la temperatura disminuyó en posición de fijación con parpadeo de $36,62{ }^{\circ} \mathrm{C}$ a 36,57 ${ }^{\circ} \mathrm{C}$ para el ojo derecho (Cuadro 4 y Figura 3), y para el ojo izquierdo de $36,10^{\circ} \mathrm{C}$ a $36,05^{\circ} \mathrm{C}$ (Cuadro 4 y Figura 4). Comparando los resultados de estos dos tipos de individuos se puede percibir que en la posición de fijación con el parpadeo normal, la temperatura de la superficie ocular del individuo sano decrece hasta obtener una diferencia de temperatura de $0,34^{\circ} \mathrm{C}$, mientras que para el paciente con ojo seco la diferencia de temperatura es de $0,05^{\circ} \mathrm{C}$.

Cuadro 3. Datos de un paciente normal en la posición de fijación con el parpadeo normal.

\begin{tabular}{|c|c|c|c|c|c|c|c|c|}
\hline \multicolumn{5}{|c|}{ Ojo derecho } & \multicolumn{4}{|c|}{ Ojo izquierdo } \\
\hline $\begin{array}{l}\text { Número } \\
\text { de datos }\end{array}$ & $\begin{array}{c}\text { Hora } \\
\text { (hh:mm:ss) }\end{array}$ & $\begin{array}{l}\text { Temperatura } \\
\left(\text { Grados }{ }^{\circ} \mathrm{C} \text { ) }\right.\end{array}$ & $\begin{array}{c}\text { Desviación } \\
\text { Estándar }\left({ }^{\circ} \mathrm{C}\right) \\
\end{array}$ & $\begin{array}{c}\text { Varianza } \\
\left({ }^{\circ} \mathrm{C}^{2}\right)\end{array}$ & $\begin{array}{c}\text { Hora } \\
\text { (hh:mm:ss) }\end{array}$ & $\begin{array}{l}\text { Temperatura } \\
\left(\text { Grados }{ }^{\circ} \mathrm{C}\right)\end{array}$ & $\begin{array}{c}\text { Desviación } \\
\text { Estándar }\left({ }^{\circ} \mathrm{C}\right)\end{array}$ & $\begin{array}{c}\text { Varianza } \\
\left({ }^{\circ} \mathrm{C}^{2}\right)\end{array}$ \\
\hline 1 & 09:42:33 p.m. & 35,09 & 0,51 & 0,26 & 09:49:02 p.m. & 34,81 & 0,60 & 0,36 \\
\hline 2 & 09:42:35 p.m. & 35,07 & 0,40 & 0,16 & 09:49:04 p.m. & 34,80 & 0,57 & 0,32 \\
\hline 3 & 09:42:37 p.m. & 35,08 & 0,36 & 0,13 & 09:49:06 p.m. & 34,77 & 0,65 & 0,42 \\
\hline 4 & 09:42:39 p.m. & 35,06 & 0,37 & 0,14 & 09:49:09 p.m. & 34,79 & 0,64 & 0,41 \\
\hline 5 & 09:42:43 p.m. & 35,06 & 0,39 & 0,15 & 09:49:11 p.m. & 34,76 & 0,68 & 0,46 \\
\hline 6 & 09:42:45 p.m. & 35,06 & 0,40 & 0,16 & 09:49:16 p.m. & 34,75 & 0,64 & 0,41 \\
\hline 7 & 09:42:47 p.m. & 35,00 & 0,40 & 0,16 & 09:49:20 p.m. & 34,75 & 0,73 & 0,53 \\
\hline 8 & 09:42:49 p.m. & 34,95 & 0,50 & 0,25 & 09:49:22 p.m. & 34,75 & 0,64 & 0,41 \\
\hline 9 & 09:42:54 p.m. & 34,92 & 0,50 & 0,25 & 09:49:24 p.m. & 34,72 & 0,65 & 0,42 \\
\hline 10 & 09:42:56 p.m. & 34,85 & 0,56 & 0,31 & 09:49:26 p.m. & 34,60 & 0,70 & 0,49 \\
\hline 11 & 09:42:58 p.m. & 34,81 & 0,56 & 0,31 & 09:49:29 p.m. & 34,61 & 0,70 & 0,49 \\
\hline 12 & 09:43:03 p.m. & 34,75 & 0,53 & 0,28 & 09:49:31 p.m. & 34,52 & 0,76 & 0,58 \\
\hline
\end{tabular}


Cuadro 4. Datos de un paciente con ojo seco en la posición de fijación con el parpadeo normal.

\begin{tabular}{|c|c|c|c|c|c|c|c|c|}
\hline \multicolumn{5}{|c|}{ Ojo derecho } & \multicolumn{4}{|c|}{ Ojo izquierdo } \\
\hline $\begin{array}{l}\text { Número } \\
\text { de datos }\end{array}$ & $\begin{array}{c}\text { Hora } \\
\text { (hh:mm:ss) }\end{array}$ & $\begin{array}{l}\text { Temperatura } \\
\left(\text { Grados }{ }^{\circ} \mathrm{C}\right)\end{array}$ & $\begin{array}{c}\text { Desviación } \\
\text { Estándar }\left({ }^{\circ} \mathrm{C}\right)\end{array}$ & $\begin{array}{c}\text { Varianza } \\
\left({ }^{\circ} \mathrm{C}^{2}\right)\end{array}$ & $\begin{array}{c}\text { Hora } \\
\text { (hh:mm:ss) }\end{array}$ & $\begin{array}{l}\text { Temperatura } \\
\left(\text { Grados }{ }^{\circ} \mathrm{C}\right)\end{array}$ & $\begin{array}{c}\text { Desviación } \\
\text { Estándar }\left({ }^{\circ} \mathrm{C}\right)\end{array}$ & $\begin{array}{c}\text { Varianza } \\
\left({ }^{\circ} \mathrm{C}^{2}\right)\end{array}$ \\
\hline 1 & 12:08:32 p.m. & 36,62 & 0,18 & 0,03 & $12: 12: 16$ p.m. & 36,10 & 0,32 & 0,10 \\
\hline 2 & $12: 08: 35$ p.m. & 36,62 & 0,18 & 0,03 & $12: 12: 19$ p.m. & 36,08 & 0,30 & 0,09 \\
\hline 3 & $12: 08: 39$ p.m. & 36,60 & 0,19 & 0,04 & $12: 12: 21$ p.m. & 36,07 & 0,31 & 0,10 \\
\hline 4 & $12: 08: 41$ p.m. & 36,58 & 0,16 & 0,03 & $12: 12: 24$ p.m. & 36,08 & 0,35 & 0,12 \\
\hline 5 & $12: 08: 43$ p.m. & 36,60 & 0,16 & 0,03 & $12: 12: 26$ p.m. & 36,06 & 0,35 & 0,12 \\
\hline 6 & $12: 08: 46$ p.m. & 36,59 & 0,15 & 0,02 & $12: 12: 28$ p.m. & 36,09 & 0,37 & 0,14 \\
\hline 7 & $12: 08: 50$ p.m. & 36,58 & 0,21 & 0,04 & $12: 12: 33$ p.m. & 36,09 & 0,33 & 0,11 \\
\hline 8 & $12: 08: 52$ p.m. & 36,57 & 0,15 & 0,02 & 12:12:35 p.m. & 36,07 & 0,33 & 0,11 \\
\hline 9 & 12:08:54 p.m. & 36,57 & 0,20 & 0,04 & $12: 12: 38$ p.m. & 36,09 & 0,34 & 0,12 \\
\hline 10 & $12: 08: 57$ p.m. & 36,56 & 0,18 & 0,03 & $12: 12: 42$ p.m. & 36,08 & 0,32 & 0,10 \\
\hline 11 & $12: 08: 59$ p.m. & 36,57 & 0,17 & 0,03 & $12: 12: 44$ p.m. & 36,06 & 0,31 & 0,10 \\
\hline 12 & 12:09:02 p.m. & 36,57 & 0,16 & 0,03 & $12: 1246$ p.m. & 36,05 & 0,33 & 0,11 \\
\hline
\end{tabular}

De igual forma se puede verificar en las Figuras 3 y 4 que el sujeto con ojo seco presenta temperaturas de la superficie ocular más altas que las temperaturas del sujeto sano, tanto para el ojo derecho como para el ojo izquierdo, a pesar de que las condiciones ambientales que se registraron mientras se tomaron las pruebas del sujeto sano (Tambiente $=23,1{ }^{\circ} \mathrm{C}$ y Hrelativa $=67 \%$ ) fueron más altas que las que se registraron durante la prueba del paciente con ojo seco (Tambiente $=22,4^{\circ} \mathrm{C}$ y Hrelativa $=66 \%$ ) fueron más altas que las que se registraron durante la prueba del paciente con ojo seco .

Figura 3. Gráficas de los resultados obtenidos para la posición de fijación con parpadeo del ojo derecho de un sujeto sano y un sujeto con ojo seco.

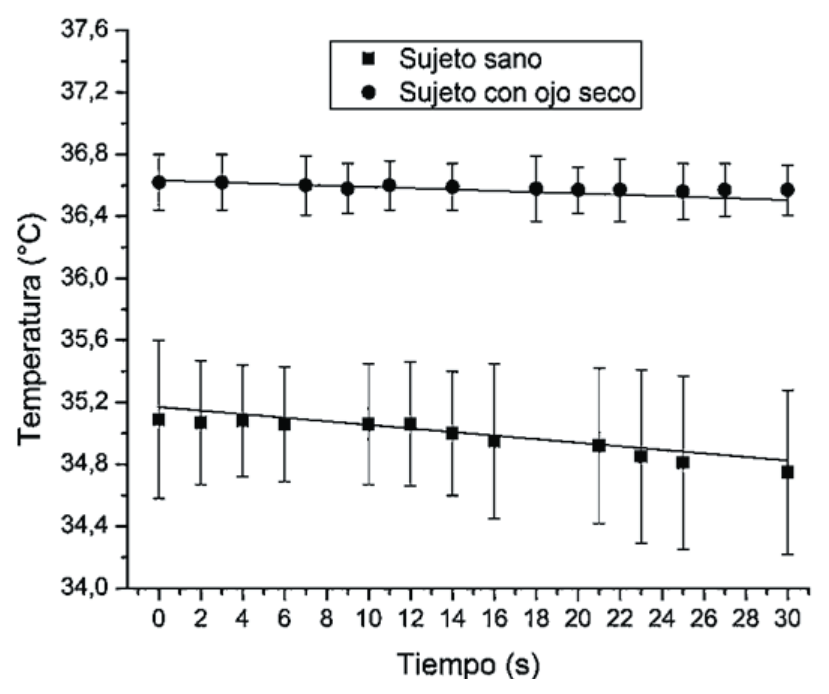

Figura 4. Gráficas de los resultados obtenidos para la posición de fijación con parpadeo del ojo izquierdo de un sujeto sano y un sujeto con ojo seco.

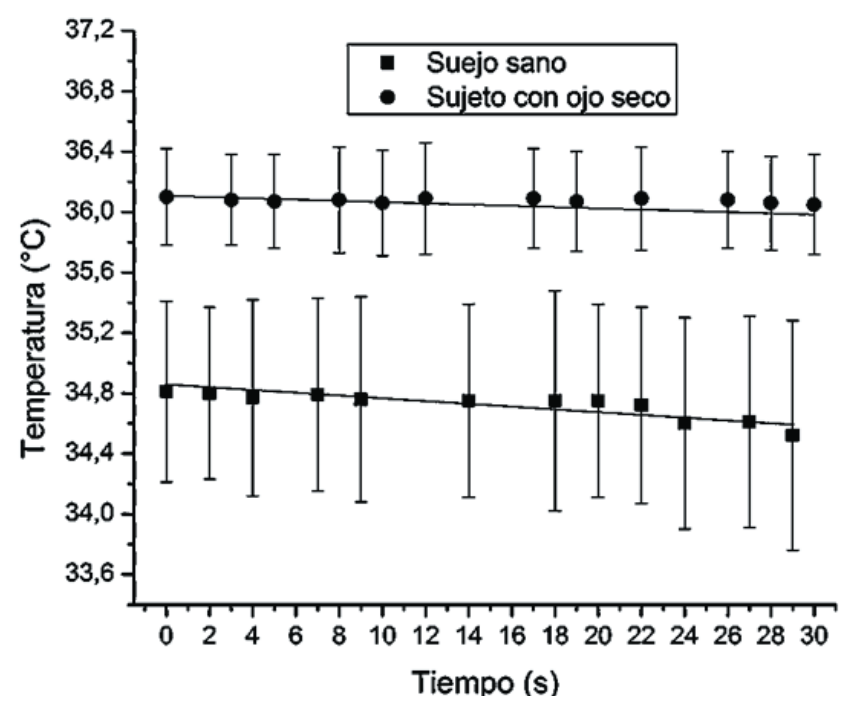

Analizando los datos de toda la muestra poblacional en que se hizo el estudio, se determinó de manera general, que la variación de temperatura de los sujetos con ojo seco (ojo derecho y ojo izquierdo) en posición de fijación con parpadeo, fue menor que la obtenida con respecto a los sujetos normales, en el cuadro 5 se pueden verificar los resultados obtenidos para los dos tipos de muestras estudiadas. 
Cuadro 5. Diferencia de temperatura en la posición de fijación con el parpadeo normal de toda la muestra.

\begin{tabular}{ccccc}
\hline \multicolumn{3}{c}{ Sujeto sano } & \multicolumn{2}{c}{ Sujeto con ojo seco } \\
\hline Sujeto & $\Delta \mathbf{T}$ Ojo derecho $\left({ }^{\circ} \mathbf{C}\right)$ & $\Delta \mathbf{T}$ Ojo izquierdo $\left({ }^{\circ} \mathbf{C}\right)$ & $\Delta \mathbf{T}$ Ojo derecho $\left({ }^{\circ} \mathbf{C}\right)$ & $\Delta \mathbf{T}$ Ojo izquierdo $\left({ }^{\circ} \mathbf{C}\right)$ \\
\hline 1 & 0,31 & 0,24 & 0,01 & 0,13 \\
2 & 0,34 & 0,29 & 0,05 & 0,05 \\
3 & 0,25 & 0,27 & 0,05 & 0,06 \\
4 & 0,23 & 0,35 & 0,05 & 0,06 \\
5 & 0,34 & 0,23 & 0,10 & 0,09 \\
6 & 0,50 & 0,36 & 0,07 & 0,03 \\
7 & 0,28 & 0,25 & 0,05 & 0,05 \\
8 & 0,42 & 0,34 & 0,10 & 0,09 \\
9 & 0,32 & 0,24 & 0,04 & 0,04 \\
10 & 0,33 & 0,29 & 0,05 & 0,07 \\
11 & 0,29 & 0,34 & 0,07 & 0,02 \\
12 & 0,30 & 0,26 & 0,10 & 0,07 \\
13 & 0,34 & 0,27 & 0,09 & 0,02 \\
14 & 0,34 & 0,33 & 0,13 & 0,09 \\
15 & 0,28 & 0,24 & 0,16 & 0,15 \\
16 & 0,36 & 0,30 & 0,16 & 0,16 \\
17 & 0,44 & 0,46 & 0,16 & 0,15 \\
18 & 0,45 & 0,15 & 0,13 \\
19 & 0,97 & 0,30 & 0,07 & 0,05 \\
\hline Media $\left({ }^{\circ} \mathbf{C}\right)$ & 0,36 & 0,45 & 0,087 & 0,077 \\
\hline Desviación & 0,38 & 0,31 & 0,045 & 0,045 \\
\hline estándar $\left({ }^{\circ} \mathbf{C}\right)$ & 0,16 & 0,072 & 0,0020 & 0,0020 \\
\hline Varianza $\left({ }^{\circ} \mathbf{C}^{2}\right)$ & 0,025 & 0,0052 & & \\
\hline
\end{tabular}

$\mathrm{Al}$ analizar la muestra total se encuentra que el rango absoluto de la disminución de temperatura de la superficie ocular de los sujetos sanos en posición de fijación con parpadeo para los ojos derechos fue de 0,23 a $0,97^{\circ} \mathrm{C}$ mientras que para los ojos izquierdos fue de 0,23 a $0,46^{\circ} \mathrm{C}$. Para los sujetos con síndrome de ojo seco se determinó una disminución de la temperatura en un rango absoluto de 0,01 a 0,16 ${ }^{\circ} \mathrm{C}$ para los ojos derechos y de 0,02 a $0,16{ }^{\circ} \mathrm{C}$ para los ojos izquierdos. Con la muestra total y tomando como referencia la variación de temperatura de los sujetos del estudio, sanos y diagnosticados con SOS, se realiza una prueba de normalidad de esos datos, encontrándose aproximadamente normales sobre todo para sujetos con ojo seco, por lo cual se decide realizar la prueba U de Mann-Whitney para datos no paramétricos con base en la cual se compararon las dos muestras (grupo de sujetos sanos y grupo con síndrome del ojo seco).

La prueba U de Mann-Whitney se realizó por medio del software para análisis de datos OriginLab 8.6, donde se establecieron las siguientes hipótesis:

Para los ojos derechos de los dos tipos de pacientes se obtuvo un $\mathrm{Z}=$ 5,40919 y un $\mathrm{p} \leq 1,45 \times 10^{-11}$ y para los ojos izquierdos un $\mathrm{Z}=5,40232$ con un $\mathrm{p} \leq 1,45 \times 10^{-11}$. Lo que quiere decir que $\mathbf{p}$ fue menor que 0,05 Por lo tanto se determinó que existe una diferencia estadísticamente significativa entre los grupos y que se puede establecer que el método diseñado e implementado puede establecer diferencias entre los dos grupos, basadas en que los sujetos que presentan una disminución de temperatura más pequeña en la etapa de fijación con parpadeo, tienen un grado de resequedad más alto comparado con los valores obtenidos para sujetos sanos.

\section{Discusión}

De manera general la temperatura de la superficie ocular de los sujetos sanos decreció rápidamente después de 30 segundos con el parpadeo normal, mientras que en los sujetos con síndrome de ojo seco, la temperatura bajo lentamente, lo cual induce a afirmar que el protocolo que se propone para la determinación cuantitativa del Síndrome de Ojo Seco se puede basar en la disminución de la temperatura promedio de la superficie corneal con respecto al tiempo, en la posición que hemos llamado de fijación con parpadeo (la mirada al frente con el parpadeo normal, durante 30 segundos).

Es interesante anotar para posteriores estudios y análisis de los datos encontrados en esta investigación, que de manera general se encuentra que los sujetos con SOS presentan una temperatura promedio mayor en su superficie corneal, al ser comparados con sujetos sanos, tal como se aprecia en las figuras 3 y 4 para el ojo derecho y ojo izquierdo de un sujeto sano y un sujeto con SOS en la etapa de fijación con parpadeo.

Para realizar una observación detallada de cada paciente se realizó un reporte donde se registran los valores de temperatura promedio con su correspondiente desviación estándar y varianza en cada una de las posiciones de fijación con y sin parpadeo, posteriormente se tienen las gráficas de cada uno de estos valores de temperatura promedio y desviación estándar tanto para el ojo derecho como para el ojo izquierdo, por último se tienen las observaciones generales donde se discuten las variaciones de temperatura con respecto al tiempo en las posiciones de fijación con y sin el parpadeo, comparados con los signos y síntomas que registró cada sujeto en su correspondiente encuesta epidemiológica.

Las condiciones ambientales (luminosidad, temperatura y humedad relativa) deben ser controladas y consignadas en el momento de realizar las mediciones; por tal razón se hace necesario utilizar un recinto en el cual estas variables se encuentren adecuadamente controladas; González M. es un trabajo realizado en 2008 con el propósito de determinar si las condiciones ambientales pueden alterar la superficie ocular de sujetos con síntomas leves de ojo seco, informa acerca de sus resultados (35): "Se evaluaron los cambios en la superficie ocular derivados de la exposición a los distintos ambientes, concluyendo que la baja humedad ambiente afecta negativamente a la superficie ocular". 
La técnica de la termografía infrarroja aplicada en seres humanos es no invasiva, en ningún momento hay una manipulación directa del sujeto, a pesar de ello el estudio con una muestra de 40 personas que perseguía demostrar la validez física del método, se implementó teniendo en cuenta el diseño de protocolos acordes con especificaciones bioéticas pues se trabaja con seres humanos.

Con base en lo anterior, en este trabajo se han propuesto los fundamentos basados en resultados experimentales, que permitirían posteriormente establecer un método para diagnosticar de manera cuantitativa el síndrome del ojo seco, mediante la técnica de la termografía infrarroja, el cual puede llegar a ser un procedimiento totalmente efectivo para el diagnóstico clínico del mismo $(5,16,33)$, ya que se basa en pruebas cuantitativas, mientras que en la actualidad el diagnóstico se hace por medio de pruebas cualitativas no muy precisas como lo es la prueba Schirmer I; sin embargo se hace necesario realizar un trabajo con una muestra poblacional mayor, con el fin de cuantificar y estandarizar de manera formal el método propuesto, de tal manera que pueda ser utilizado para el diagnóstico y seguimiento clínico del SOS.

\section{Agradecimientos}

A COLCIENCIAS, Departamento Administrativo de Ciencia Tecnología e Innovación por su programa de jóvenes Investigadores e Innovadores 2013-2014 y a la Universidad Tecnológica de Pereira por apoyar dicho programa y apoyar el proyecto "Protocolo de diagnóstico del síndrome del ojo seco a partir de la fotografía termográfica”.

\section{Conflicto de interés}

Los autores declaran no tener conflictos de interés.

\section{Referencias}

1. Acharya R, Chang Yee G, Tin Jian H, and Kagathi M. Analysis of normal human eye different age groups using infrared images. Journal of Medical Systems. 2009; 33:207-213.

2. Gálvez T, Lou R, and Andreu E. Ojo seo: diagnóstico y tratamiento. Información Terapéutica del Sistema Nacional de Salud. 1998; 22(5):117-122.

3. Victorero Y, Martínez Z, Triana I, Sablón R, Hernández C, and Morell Z. Características clínico-epidemiológicas del síndrome de ojo seco en el Policlínico Federico Capdevilla durante el año 2010. Centro oftalmológico Enrique Cabrera. 2013; 19(1).

4. Dapena M and Lavín C. Diagnostico SOPV In: Dapena M and Lavín C. Trastornos visuales del ordenador. Madrid: Producciones Pantúas; 2005.p. 115-133.

5. Fujishima H, Toda I, Yamada M, Sato N, and Tsubota K. Corneal temperature in patients with dry eye with dry eye evaluated by infrared radiation thermometry. British Journal of Ophthalmology. 1996; 80:29-32.

6. Tsubota K. Tear dynamics and dry eye. Progress in Retinal and Eye Research. 1998; 17(4):565-596.

7. Tan J, Ng E, Acharya R, and Chee C. Infrared thermography on ocular surface temperature: A review. Elsevier, Infrared Physics \& Technology. 2009; 52:97-108.

8. Colin H. Medical thermography. IEE Proceedings. 1987; 134(2): 225-236.

9. Jones B. A Reappraisal of the use of infrared thermal image analysis in medicine. IEEE Transactions on medical imaging. 1998; 17(6):1019-1027.
10. Szentkuti A, Kavanagh H, Grazio S. Infrared thermography and image analysis for biomedical use. Periodicum Biologorum. 2011; 113(4):385-392.

11. Morgan P, Soh M, Efron N. Corneal surface temperature decreases with age. Contacs Lens and Anterior Eye. 1999; 22(1):11-13.

12. Arora N, Martins D, Ruggerio D, Tousimis E, Swistel A, Osborne $\mathrm{M}$, et al. Effectiveness of a noninvasive digital infrared thermal imaging system in the detection of breast cancer. The American Journal of Surgery. 2008; 196:523-526.

13. Qi $\mathrm{H}$, and Diakides $\mathrm{N}$, Infrared imaging in medicine. Electrical and Computer Engineering Departament, University of Tennessee. 2001; 1-10.

14. Tan L, Cai Z, Lai N. Accuracy and sensitivity of dynamic ocular thermography and inter-subjects ocular surface temperature (OST) in Chinese young adults. Contact Lens \& Anterior Eye. 2009; 32:78-83.

15. Shiloh R, Portuguese S, Bodinger L, Katz N, Sigler M, Hermesh H, et al. Increased corneal in drug-free male schizophrenia patients. Elsevier, European Neuropsychopharmacology. 2003; 13:49-52.

16. Su T, Hwa C, Lui P, Wu M, Chang D, Su P, et al. Noncontact detection of dry eye using a custom designed infrared thermal image system. Journal of Biomedical Optics. 2011; 16(4):1-6.

17. E. Rinenberg, Centro Oftalmológico Integral. 2004. [Online] Available: http://www.centro-rinenberg.com.ar/ojoseco.html. [Accessed Septiembre 2014].

18. Cho P and Yap M. Effect of contact lens wear on the tears of Hong Kong Chinese. Journal of the British Contact Lens Association. 1995; 18(3):87-94.

19. Outeriño E. Prevalencia del ojo seco en el área sanitaria de $O$ Salnés. Trabajo de grado de Doctor en Medicina y Cirugía. Santiago de Compostela: Universidad Santiago de Compostela, Facultad de Medicina, Departamento de Cirugía. 2008.

20. Maderos $M$, Menese $M$, Landrían $B$, and Zayas $Y$. Comportamiento clínico y epidemiológico del ojo seco en el servicio de oftalmología del Hospital Provincial de Ciego de Ávila. Revista de Oftalmología Misión Milagro. 2008; 2(2).

21. Battaglini P. Fisiología de la visión In: Conti F. Fisiología Médica. México D.F: MacGraw-Hill Interamericana Editores, S.A; 2010. Pp. 379-402.

22. Garg A. Fisiopatología de la película lagrimal In: Garg A, Sheppard J, Donnenfeld E, Meyer D, Mehta C. Ojo seco y otros trastornos de la superficie ocular. Diagnóstico y tratamiento en Xerodacriología. Médica Panamericana; 2008. Pp. 1-28.

23. Fluke Corporation and The Snell Group. Introducción a los principios de la Termografía. Paises Bajos: American Technical publishers; 2009.

24. Fluke Corporation. Manual de uso Thermal Imager Ti32. USA: 2009.

25. Buccella C, Satis V, and Feliziani M. Prediction of temperature increase in human eye due to RF sources. IEEE Transactions on electromagnetic compatibility. 2007; 49(4):825-833.

26. Lahiri B, Bagavathiappan S, Joyakumar T and Philip J. Medical applications of infrared thermography: A review. Elsevier, Infrared Physics \& Technology. 2012; 55:221-235.

27. Testo A G. Termografía, guía de bolsillo. Teoría, Aplicación Práctica, Consejos y Trucos. 2008.

28. Asociación Médica Mundial. Declaración de Helsinki. Principios éticos para las investigaciones médicas en seres humanos. Junio 1964, enmendada 2008. [Online] Available:http://www.wma.net/ es/30publications/10policies/b3/17c_es.pdf.[Accessed Marzo 2015]. 
29. Ministerio de Salud. República de Colombia. Resolución No 008430 de 1993, Titulo II, Capitulo 1, de los aspectos éticos de la investigación en seres humanos. Octubre de 1993. [Online] Available:http://www.unisabana.edu.co/fileadmin/Documentos/ Investigacion/comite_de_etica/Res__8430_1993_-_Salud.pdf. [Accessed: Marzo 2015].

30. Purslow C, Wolffsohn J and Rubido J. The effect of contact lens wear on dynamic ocular surface temperature. Contacts Lens \& Anterior Eye. 2005; 28: 29-36.

31. Kessel L, Johnson L, Arvidsson H and Larsen M. The relationship between Body and Ambient Temperature and Corneal Temperature. Investigative Ophthalmology \& Visual Science. 2010; 51(12): 6593-6597.

32. Laboratorio de Producción. Facultad de Ingeniería Industrial. Escuela Colombiana de Ingeniería Julio Garavito. Protocolo de Iluminación. Laboratorio de condiciones de trabajo. 2008.

33. Morgan P, Tullot A and Efron N. Ocular surface cooling in dry eye - A pilot study. Journal of the British Contact Lens Association. 1996; 19(1):7-10.

34. Morgan P, Soh M and Efron N. Potential Applications of Ocular Thermograaphy. Optometry and vision science. 1993; 70(7):568576.

35. González M. Alteración de la superficie ocular tras la exposición a un ambiente adverso creado en una cámara de ambiente controlado (CAC). Trabajo de grado doctoral en Ciencias de la Visión. Valladolid: Universidad de Valladolid. Instituto Universitario de Oftalmobiología Aplicada (IOBA), 2008 